Int. J. Dev. Biol. 49: 443-448 (2005)

doi: $10.1387 /$ ijdb.051984lp

Short Communication

\title{
Genetic interaction of Gli3 and Alx4 during limb development
}

\author{
LIA PANMAN*,a, THIJS DRENTH ${ }^{\mathrm{b}}$, PASCAL TEWELSCHER ${ }^{\mathrm{c}}$, AIMÉE ZUNIGA ${ }^{1}$ and ROLF ZELLER ${ }^{1}$ \\ Department of Developmental Biology, Utrecht University, Utrecht, The Netherlands and ${ }^{1}$ Developmental Genetics, DKBW Centre for \\ Biomedicine, University of Basel Medical School, Basel, Switzerland.
}

\begin{abstract}
The Gli3 and Alx4 transcriptional regulators are expressed in the anterior limb bud mesenchyme and their disruption in mice results in preaxial polydactyly. While the polydactylous phenotype of Alx4 deficient limb buds depends on SHH, the one of Gli3 deficient limb buds is completely independent of $\mathrm{SHH}$ signalling, suggesting that these genes act in parallel pathways. Analysis of limb buds lacking both Gli3 and Alx4 now shows that these two genes interact during limb skeletal morphogenesis. In addition to the defects in single mutants, the stylopod is severely malformed and the anterior element of the zeugopod is lost in double mutant limbs. However, limb bud patterning in $\mathrm{Gli3}^{\% /-} ; A / \mathrm{X}^{\%} /$ double mutant embryos is not affected more than in single mutants as the expression domains of key regulators remain the same. Most interestingly, the loss of the severe preaxial polydactyly characteristic of Gli3-/- limbs in double mutant embryos establishes that this type of polydactyly requires Alx4 function.
\end{abstract}

KEY WORDS: Hox gene, limb development, preaxial polydactyly, radius, SHH, tibia

The semi-dominant mouse mutations Extra-toes $(X t)$ and Strong's Luxoid ( $L s t)$, are loss-of-function mutations disrupting the zincfinger encoding transcription factor Gli3 (Schimmang et al., 1992; Hui and Joyner, 1993) and the aristaless-related homeobox gene Alx4 (Qu et al., 1998; Takahashi et al., 1998), respectively. In the homozygous state, both mouse mutations cause pleiotropic and lethal congenital malformations with distinct preaxial limb polydactylies. In both mutants, an anterior ectopic Sonic Hedgehog (SHH) signalling centre is established in addition to the one in the posterior limb bud mesenchyme (Masuya et al., 1995; Buscher et al., 1997; Qu et al., 1997). SHH is a morphogen produced by the polarizing region (or ZPA) in the posterior limb bud and is essential for controlling antero-posterior patterning of the limb skeleton (zeugopod and autopod; reviewed by Zeller, 2004). Only one anterior zeugopodal element and digit develop in limbs of embryos lacking SHH function (Chiang et al 2001; Kraus et al., 2001). Interestingly, the polydactylous phenotypes caused by the mutations in Gli3 and $A / \times 4$ differ with respect to their dependence on $\mathrm{SHH}$ signalling. The polydactyly of Gli3 deficient limbs is $\mathrm{SHH}$ independent as limbs lacking both $G / i 3$ and $S h$ display a polydactyly identical to Gli3 single mutants. In contrast, the one caused by disrupting $A / x 4$ is $\mathrm{SHH}$ dependent as only one digit forms in limbs lacking both $A / x 4$ and Shh (for details see te Welscher et al., 2002b). Indeed, Gli3 functions first in a mutually antagonistic interaction with $d$ Hand, which seems to pre-pattern the limb bud mesenchyme already prior to Shh activation (te Welscher etal., 2002a). In contrast, $A / \times 4$ does not seem to function during this early determinative phase upstream of $\mathrm{SHH}$ mediated limb bud patterning. Additional striking differences between the two mutant limb phenotypes (Qu et al., 1997; Zuniga et al., 1999; te Welscher et al., 2002b) are a likely consequence of their differential functions: anterior expansion of the normally posteriorly restricted expression domains of Gremlin and 5\% Hoxd genes is apparent from the earliest limb bud stages onwards in Gli3 deficient limb buds. In contrast, small anterior ectopic expression domains appear only much later in $A / x 4$ deficient limb buds. Furthermore, $A / x 4$ expression is reduced in $G / i 3$ deficient limb buds, indicating that G/i3 acts genetically upstream of $A / x 4$ (te Welscher et al., 2002a). However, the remaining, more proximally restricted $A / x 4$ expression in Gli3 $\%$ mutant limb buds also indicates that Alx4 may function at least in part independent of Gli3. Taken together, these and other studies establish that Gli3, but not

Abbreviations used in this paper: $\mathrm{Xt}$, extra toes.

a) Present address: Ludwig Institute for Cancer Research, Department of Cell and Molecular Biology, Karolinska Institute, Box 240, S-17177 Stockholm, Sweden.
b) Present address: INSERM U.623-IBDM, Campus de Luminy-Case 907, F-13288 Marseille Cedex 09, France.
c) Present address: EMBL Monterotondo, Mouse Biology Unit, Via Ramarini 32, 00016 Monterotondo, Italy.

*Address correspondence to: Dr. Lia Panman. Ludwig Institute for Cancer Research, Department of Cell and Molecular Biology, Karolinska Institute, Box 240, S-17177 Stockholm, Sweden. Fax: +46-8-332-812. e-mail: lia.panman@licr.ki.se 
A
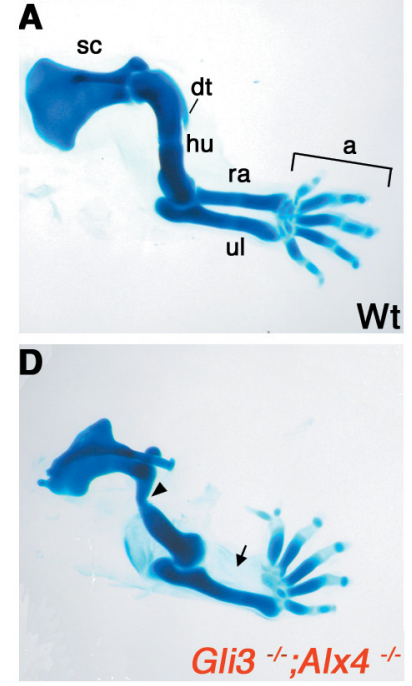

G

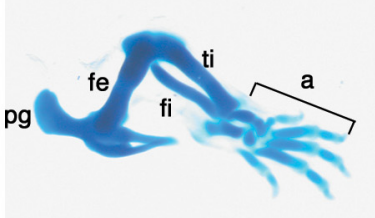

$J$

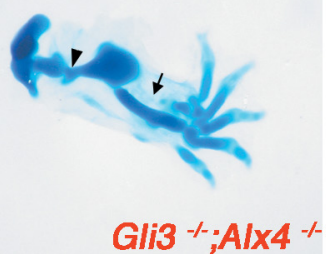

B
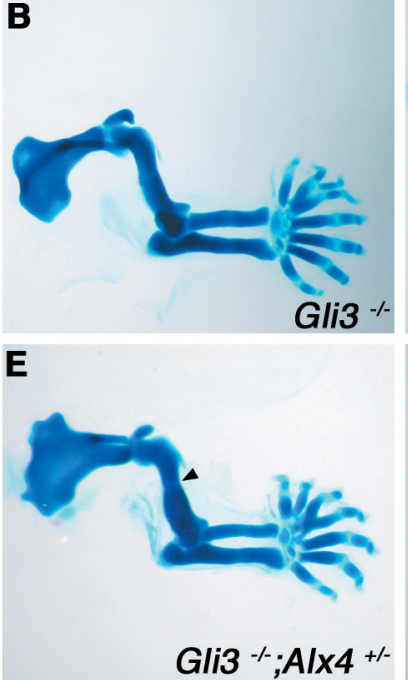

H

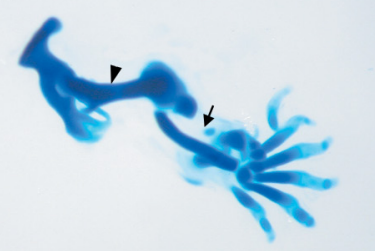

Gli3

K

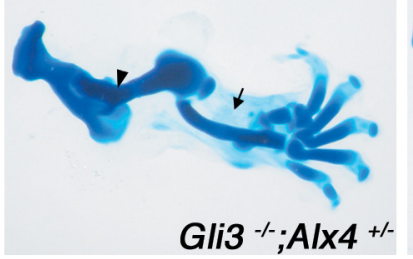

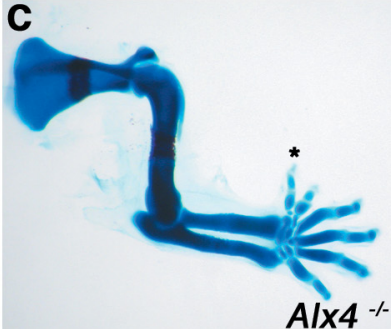

$\mathbf{F}$

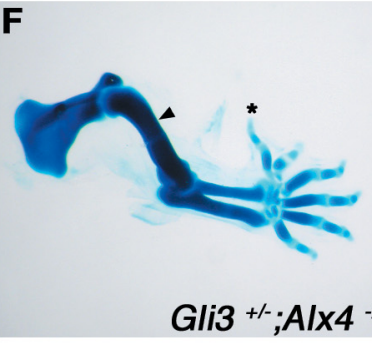

I

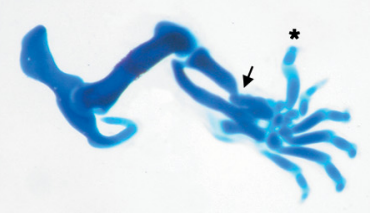

Alx4

Fig. 1. Skeletal preparations of limbs of single and compound mutant embryos lacking Gli3 and/or Alx4 at gestational day E14.5. (A-F) forelimbs, (G-L) hindlimbs, cartilage appears blue as stained by alcian blue. As ossification is just being initiated, none or only few ossification centres become apparent as darker areas (alizarin red). (A) Wild-type forelimb. (B) Gli3--- homozygous forelimb. Note the preaxial polydactyly and loss of digit identity. (C) Alx4--- homozygous forelimb. Asterisk indicates a duplicated preaxial digit 2. (D) Gli3-/-; Alx4--double homozygous forelimb. Arrowhead points to the malformed humerus. Arrow points to the anterior zeugopodal region lacking the radius. Note the duplication of the distal phalange of the most anterior digit. (E) Gli3-/-; Alx4+/- mutant forelimb, arrowhead indicates the malformed humerus. The preaxial polydactyly of the autopod is similar to the one of a forelimb lacking Gli3 alone. (F) Gli3+/-; Alx4-/mutant forelimb. Asterisk indicates the duplicated preaxial digit 2. Arrowhead points to the humerus lacking the deltoid tuberosity. Note that the phenotype of the autopod is identical to the one of a forelimb lacking Alx4 alone. (G) Wild-type hindlimb. (H) Gli3-/- homozygous hindlimb. Arrow points to the rudimentary tibia. (I) Alx4-/- homozygous hindlimb. Arrow points to the deformed tibia. Asterisk indicates the duplicated preaxial digit 2. (J) - Gli3---; Alx4-/- double homozygous hindlimb. Arrow points to the region lacking the tibia. Arrowhead indicates the malformed femur. (K) Gli3-/-; Alx4+/mutant hindlimb. The femur (arrowhead) and tibia (arrow) are affected. (L) Gli3+/-; Alx4-/- mutant hindlimb. Arrow points to the malformed tibia. Asterisk points to the duplicated preaxial digit 2. All panels are oriented with anterior to the top and distal to the right. a, autopod; $d t$, deltoid tuberosity; hu, humerus; ra, radius; sc, scapula; ul, ulna; fe, femur; Gli3 $^{+-} ;$AlX4 $4^{-/-}$fi, fibula; pg, pelvic girdle; ti, tibia.

Alx4, acts initially up-stream of $\mathrm{SHH}$ to polarise the nascent limb bud mesenchyme (Masuya et al., 1995; Buscher et al., 1997; te Welscher et al., 2002a). Subsequently, SHH mediated inhibition of Gli3 protein processing produces a Gli3 repressor gradient that has been proposed as essential to antero-posterior limb bud patterning (Wang et al., 2000). During progression of limb bud development, Alx 4 becomes critical to suppress anterior ectopic Shh expression (Qu et al., 1997). To uncover possible genetic interactions of Gli3 and $A / x 4$, we have generated $G / i 3$ and $A / x 4$ compound mutant embryos for analysis. Up to gestational day 14.5 (E14.5), all genotypes are recovered from litters at expected ratios (Fig. 1 and data not shown), but by E16.5 no double homozygous embryos

TABLE 1

\section{SKELETAL PHENOTYPES}

\begin{tabular}{|c|c|c|c|c|c|}
\hline & G/i3/ & $A / \times 4^{/}$ & Gli3/ $; A / x 4^{+/}$ & Gli $3^{+/} ; A / \times 4^{-}$ & Gli3 $^{-/-} ; A l \times 4^{-/-}$ \\
\hline Humerus & wild-type & wild-type & slightly abnormal & slightly-abnormal & malformed \\
\hline Radius & wild-type & wild-type & wild-type & wild-type & complete aplasia \\
\hline Femur & slightly abnormal & wild-type & slightly abnormal & wild-type & malformed \\
\hline Tibia & malformed & malformed & complete aplasia & malformed & complete aplasia \\
\hline Digit number & $\sim 8$ & 6 & $\sim 7$ & 6 & $5(6)$ \\
\hline
\end{tabular}

could be collected due to embryonic lethality (data not shown). The skeletons of four embryos lacking both G/i3 and $A / x 4$ at E14.5 were analysed and all double homozygous limbs display skeletal defects that are distinct from the ones of all other genotypes (Fig. 1, 2 and data not shown; for summary see Table 1). In addition, severe craniofacial clefting of the nose region affects these embryos by E14.5 (data not shown).

\section{Skeletal Phenotypes of Forelimbs}

The scapula and stylopod of Gli3-/-; Alx4-/- double homozygous forelimbs are malformed (Fig. 1D; arrowhead points to malformed humerus) and the latter lacks a well-formed deltoid tuberosity. In contrast, the humerus of G/i3 (Fig. 1B) and $A / x 4$ (Fig. 1C) single mutant embryos appears normal, while additional inactivation of either one Gli3 or $A / \times 4$ allele causes slight malformation of the humerus (arrowheads Fig. 1E, F). These results are indicative of a dose-dependent requirement of these two genes during humerus morphogenesis. In contrast to all other genetic combinations (Fig. $1 \mathrm{~A}-\mathrm{C}, 1 \mathrm{E}, \mathrm{F}$ and data not shown), the zeugopod of Gli3-1- ; Alx4-\% double homozygous forelimbs 
lacks the radius (arrow, Fig. 1D), while the ulna forms correctly. Finally, the autopod of double homozygous limbs is distinct from all other mutant combinations (Fig. 1D and Fig. 2A-F, for details see below).

\section{Skeletal Phenotypes of Hindlimbs}

Similar to the forelimbs, the pelvic girdle and femur of Gli3-/- ; $A / x 4$-/- double homozygous hindlimbs are severely malformed (arrowhead, Fig. 1J). The femur of Gli3- $\%$; $A 1 \times 4+1-$ compound mutant hindlimbs (arrowhead Fig. $1 \mathrm{~K}$ ) is affected similar to Gli3-/- limbs (arrowhead in Fig. $1 \mathrm{H}$ ), while it is normal in embryos lacking $A / x 4$ (Fig. 1I, L). In contrast to forelimbs, the tibia is missing in both Gli3-/- ; $A / x 4-/-$ and Gli3-/-; $A / x 4+1-$ limbs (arrows in Figs. $1 \mathrm{~J}, \mathrm{~K}$ ) and its medial part is deleted in G/i3-/- single (arrow, Fig. $1 \mathrm{H}$ ) and $G / 3+/-; A / x 4-/-$ compound mutant hindlimbs (arrow, Fig. $1 \mathrm{~L}$ ). Furthermore, the tibia is also deformed in $A / x 4-\%$ single mutant embryos (Fig. 1I), which shows that both genes are required and apparently fulfil only partially overlapping functions during tibia morphogenesis. Development of the hindlimb autopod (Fig. 1G-L) is affected similarly to forelimbs. conclusions of recent studies (Ahn and Joyner, 2004; Harfe et al., 2004), the posterior 3 digits retain distinct identities, while the five anterior-most digits lack defined identity (as indicated by question marks in Fig. 2B). Rather unexpectedly, complete inactivation of Alx4 and Gli3 (Fig. 1D and Fig. 2D) reduces digit numbers from eight (Fig. 2B, E) to five or six (Fig. 1D, 2D, 2J). The posterior three digits retain at least partial posterior identities (similar to G/i3-/- ; compare Fig. 2D to $2 \mathrm{~B}$ ), while the anterior two digits display no defined identities. Most importantly, these results establish that the severe preaxial polydactylous limb phenotype of Gli3 deficient embryos (Fig. 1B, 2B) depends on Alx4 function. While digits 2 to 5 are patterned under the influence of $\mathrm{SHH}$-mediated graded inhibition of Gli3 repressor formation, the most anterior digit 1 and the anterior zeugopod (radius and tibia) are specified by a so far unknown other mechanism (reviewed by Zeller, 2004). The results of our study now suggest that interaction of the Alx4 transcription factor with the Gli3 (repressor) protein may be involved in specifying the anterior-most limb skeletal elements (digit 1, radius and tibia). In support of this proposal, the characteristic duplicated preaxial digit observed in forelimbs lacking $A / \times 4$ but not Gli3 (Fig.

\section{Phenotypes of the Autopod}

An additional preaxial digit forms in $A / \times 4 \%$ limbs (Fig. 1C, 2C) in comparison to wildtype limbs (Fig. 1A, 2A), irrespective of the presence of either both (Fig. 1C, 2C; see also Qu et al. 1997) or only one functional Gli3 allele (Fig. 1F, 2F). In contrast, the autopods of Gli3 deficient limbs display severe preaxial polydactylies with up to eight digits (Fig. 1B, 2B). In agreement with the

Fig. 2. Skeletal phenotypes forelimbs of different genotypes at E14.5 and E12.5. (A-F) forelimbs at gestational day E14.5. (A) Wild-type control. (B) Gli3 deficient forelimb. Note the preaxial polydactyly, loss of anterior identities and webbing. (C) Alx4 deficient forelimb. Note the additional preaxial digit. (D) Gli3 -/- ; Alx4-/ forelimb. Note the lack of the radius (arrow), duplicated of the distal phalange of the most anterior digit and general webbing. (E) Gli3-/-; Alx4+/- forelimb. Note the preaxial polydactyly and webbing similar to Gli3 -/ forelimbs. (F) Gli3+/-; Alx4-/- forelimb. Note the additional preaxial digit. Arrows in (A), $(C)$ and $(F)$ indicate the interdigital areas separating the digits through apoptosis. Arrowheads in (B), (D) and (E) indicate webbing in forelimbs of embryos lacking Gli3. Digits are numbered according to their apparent identities. Question marks indicate unclear identities. (G-L) Alcian green staining of the forming cartilage elements in forelimbs at E12.5. (G-I), (L) The cartilage primordia of the radius has formed in (G) wild-type, (H) Gli3-/-, (I) Alx4-/- and (L) Gli3+/-; Alx4-/- mutant forelimbs. (K) The radius primordia is only weakly apparent in Gli3-/-; Alx4+/- mutant forelimbs (arrow). (J) The radius primordia is lacking in Gli3-/-; Alx4-/- double mutant forelimbs (arrow). All forelimbs are oriented with anterior to the top and distal to the right. hu, humerus; ra, radius; ul, ulna.
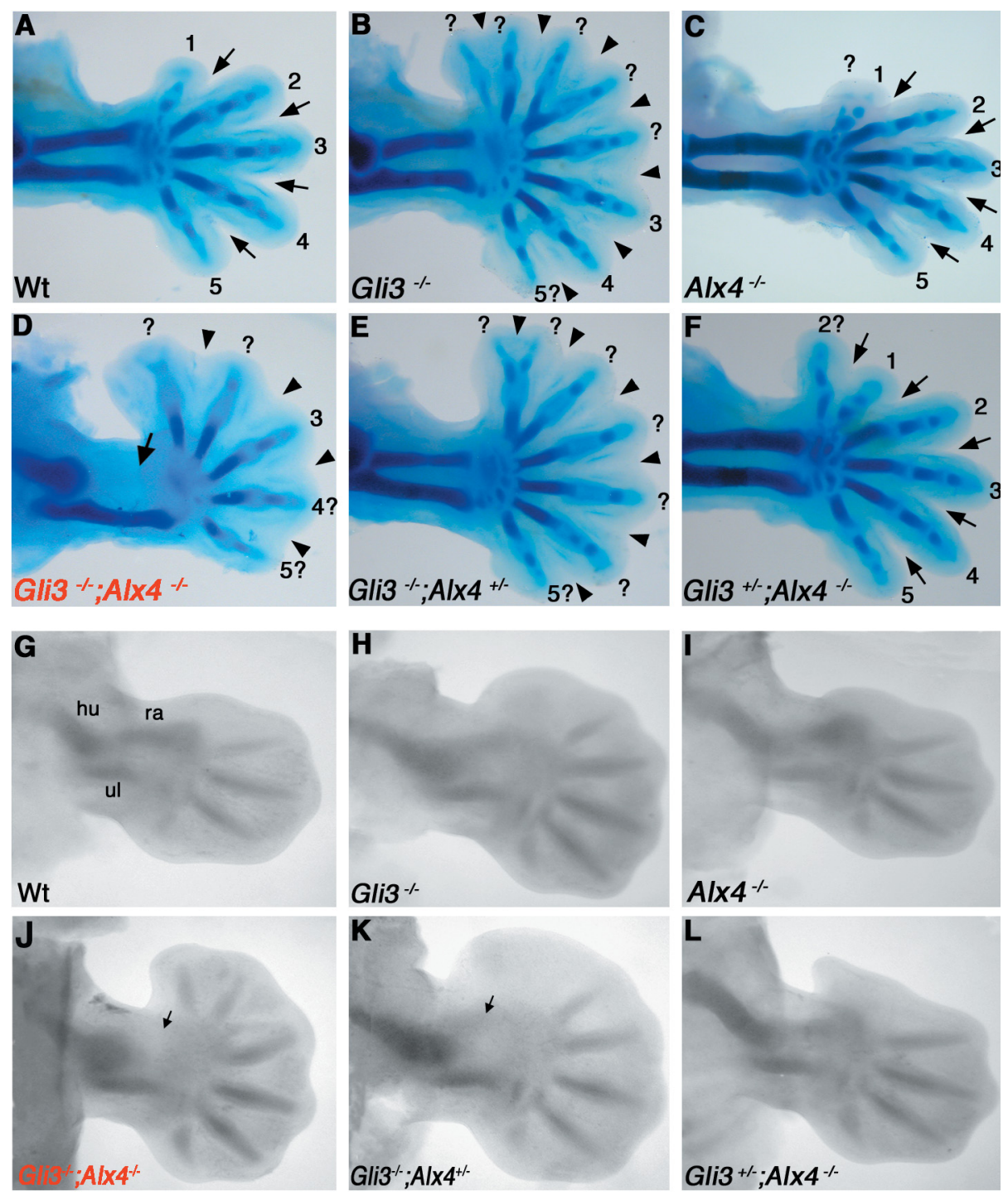

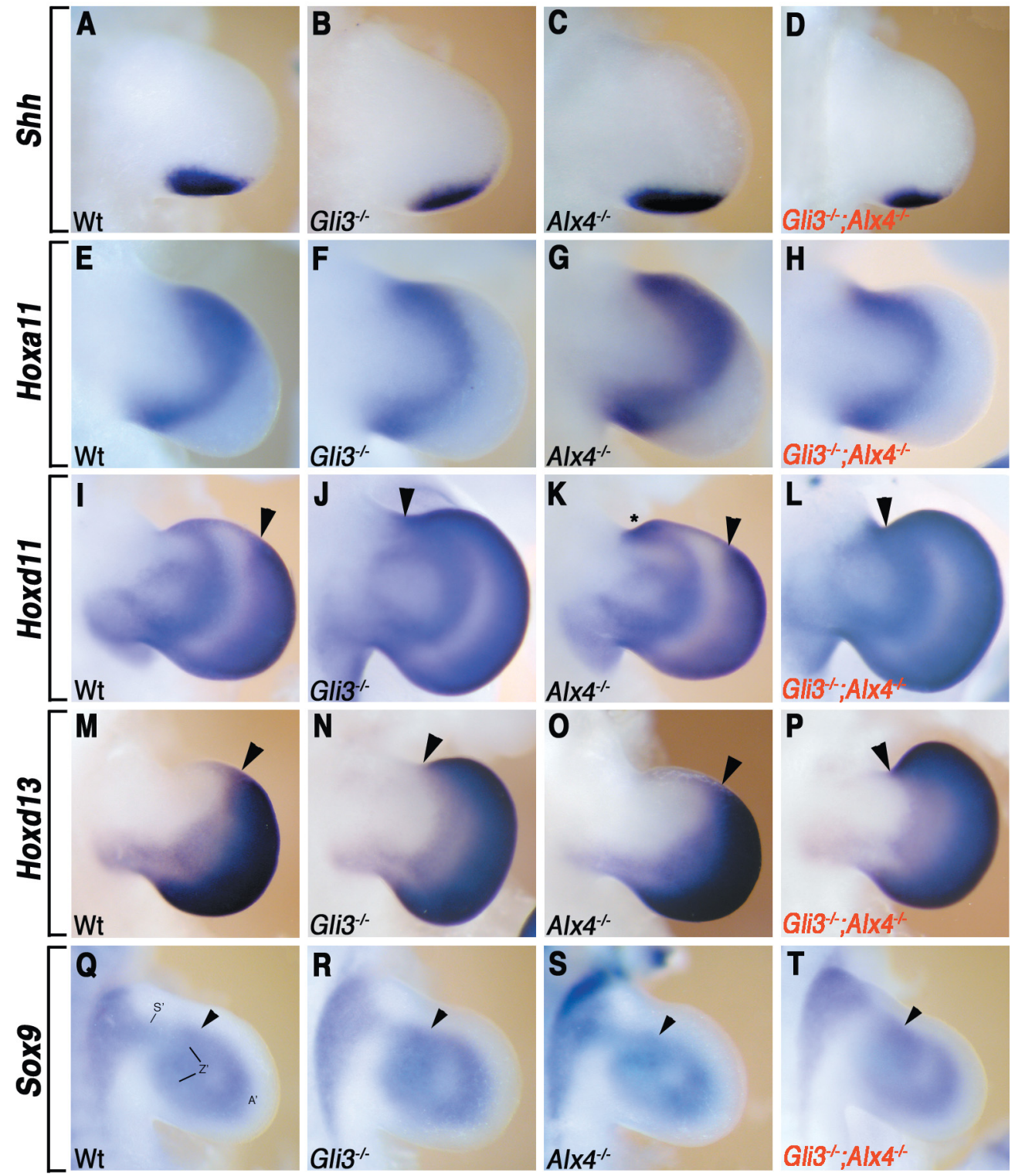

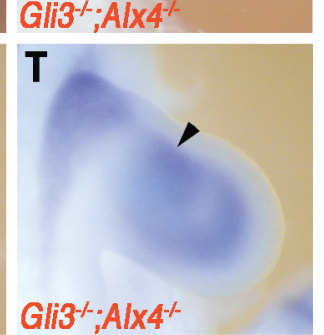

Fig. 3. Molecular analysis of key regulators of limb bud patterning. (A-D) Shh expression in wild-type and mutant forelimb buds (E10.75). At this stage, there are no significant differences in Shh expression between wild-type $(\mathbf{A})$, single $(\mathbf{B}, \mathbf{C})$ and double homozygous mutant limb buds (D). Anterior ectopic Shh expression is detected much later in mutant limb buds (data not shown; Buscher et al., 1997; Masuya et al., 1995; Qu etal., 1997). (E-H) Expression of Hoxa11 in wild-type and mutant forelimb buds (E11.5). Note that the Hoxa11 expression domain in Gli3-/; Alx4-/- limb buds (H) is identical to the one in Gli3-/- limb buds (F). (I-L) Hoxd11 expression in wild-type and mutant forelimb buds (E11.75). Again the Hoxd11 expression domain in Gli3-/-; Alx4-/- (L) and Gli3-/- limb buds (J) are similar. (M-P) Hoxd13 expression in the distal limb mesenchyme of wild-type and mutant limb buds (E11.75). The Hoxd13 expression in Gli3-/-; Alx4-/- limb buds (P) again resembles the one of Gli3-/- limb buds (N). Arrowheads in (I-P) indicate the anterior boundaries of the distal (autopod) expression domains. Asterisk in (K) indicates the ectopic anterior domain of Hoxd11 expression (Qu et al., 1997). (Q-T) Sox9 expression in wt and mutant limb buds (E11). Sox9 marks pre-cartilaginous condensations of mesenchymal cells. No significant differences are apparent. Arrowheads point to the approximate position of the condensations giving rise to the primordia of the radius. $S^{\prime}$ : prospective stylopod; Z': prospective zeugopod; $A^{\prime}$ : prospective autopod. All limb buds are oriented with anterior to the top and distal to the right.
1C, F and Fig. 2C, F) is also absent in most double mutant forelimbs (Fig. 1D, 2D). However, the distal most phalange of the most anterior digit is often duplicated (Fig. 1D, 2D). In contrast to inactivation of both $A / \times 4$ alleles (arrows, Fig. 2C, F), the loss of G/i3 functions causes interdigital webbing (arrowheads, Fig. 2B, E) by blocking interdigital apoptosis (Macias etal., 1999). This interdigital webbing is not altered by concurrent removal of $A / x 4$ in double homozygous limbs (Fig. 2D), which points to a non-redundant and essential function of Gli3 in interdigital apoptosis.

\section{Phenotypes of the Zeugopod}

The most severe additional limb skeletal defect observed in embryos lacking both G/i3 and $A / x 4$ in comparison to single homozygous mutants is the loss of the anterior zeugopodal element (radius and tibia, Fig. 1). Analysis of advanced limb bud patterning stages (E12.5, Fig. 2G-L) shows that the cartilage model giving rise to the radius does not form in double homozygous limb buds (arrow, Fig. 2J). Unexpected from the analysis of older stages (Fig. 1E, 2E), the radius primordia is also less prominent in Gli3-/-; $A / \times 4+1-$ compound mutant limb buds at this stage (arrow, Fig. 2K) than in wild-type (Fig. 2G) and the other genotypes (Fig. 2H, I, L and data not shown). These results indicate that formation of the radius is delayed in limbs lacking $G / i 3$ and one copy of $A / \times 4$ (Fig. 2K), but catches up during its subsequent development (Fig. 1E, 2E) in contrast to the resulting aplasia in double homozygous forelimbs (Fig. 1D, 2D, 2J).

\section{Normal Expression of Molecular Markers of Limb Bud Pattern- ing}

As in particular formation of anterior skeletal elements is disrupted in double homozygous limbs, the expression of relevant key regulators was analysed. Shh remains expressed normally in the posterior mesenchyme of single and double homozygous limb buds (Fig. 3A-D). No precocious anterior ectopic Shh expression is observed in double homozygous limb buds (Fig. 3D) as is the case later in single mutant limb buds (Masuya et al., 1995; Buscher 
etal., 1997; Qu et al., 1997). Genetic analysis has established that the paralogous Hoxa11 and Hoxd11 genes interact to specify the ulna and radius (Davis et al., 1995) and proximal miss-expression of Hoxd13 inhibits zeugopod morphogenesis (Goff and Tabin, 1997). Therefore, these three Hox genes are good candidates to detect alterations affecting zeugopod patterning. However, expression and distribution of neither Hoxa11 nor Hoxd11 transcripts (Fig. $3 \mathrm{H}, \mathrm{L}$ ) is significantly altered in G/i3-/-; $A / \times 4-/-$ double homozygous forelimb buds in comparison to Gli3 deficient counterparts (Fig. 3F, 3J). Furthermore, no significant changes in Hoxd13 expression are observed in Gli3-/ ; $A / \times 4 \%$ - double homozygous limb buds (Fig. 3P) in comparison to single mutant and wild-type limb buds (Fig. 3M-O). Therefore, the absence of the radius is not paralleled by consistent changes in the expression patterns of these three Hox genes during stages of limb bud patterning. In particular, the anterior limits of the Hoxd11 and Hoxd13 expression domains in the distal limb bud mesenchyme of Gli3-/ ; $A / \times 4-\%$ - embryos (arrowheads, Fig. 3L, P) are identical to the ones in Gli3-/- limb buds (arrowheads, Fig. 3J, N) and differ significantly from the ones in $A / \times 4 \%$ and wild-type limb buds (arrowheads, Fig. 3I, K, M, O). These results indicate that G/i3, but not $A / x 4$, is required to regulate the anterior limits of the Hoxd11 and Hoxd13 expression domains. The establishment of these expression domains is regulated by Gli3 already during onset of limb bud development (Zuniga and Zeller, 1999). Finally, the Sox9 transcription factor is expressed by the condensing mesenchymal cells that prefigure the limb cartilage elements (Wright etal., 1995). Analysis of Sox 9 expression reveals that the mesenchymal condensations are induced apparently normal in Gli3-/- ; AlX4-/double homozygous limb buds (Fig. 3T; compare to Fig. 3Q-S). Taken together, the results shown in Figure 3 indicate that the patterning events directing initiation of mesenchymal condensations and cartilage models, in particular the anterior limb bud, occur to the same extent in G/i3-/- ; $A / x 4-/-$ and Gli3 - / limb buds.

The present study reveals overlapping functions of Gli3 and Alx4 in formation of the radius, while truncations (but not complete loss) of the tibia have also been previously reported for $A / x 4-/-$ and Gli3-/- single mutant limbs (Johnson, 1967; Qu et al., 1998). In forelimb buds lacking both Fgf4 and Fgf8, the skeletal progenitors forming the zeugopodal condensations are reduced, which most likely directly causes loss of the radius (Sun et al., 2002). In contrast, the apparently normal Sox9 distribution in G/i3-/- ; $A / \times 4$-/- double mutant limb buds indicates that the skeletal progenitor population is not reduced and induction of the mesenchymal condensations occurs apparently normal. Defects affecting the zeugopod are also observed in a variety of other mouse mutations, in particular those affecting 5'Hoxd and 5'Hoxa genes (Zakany and Duboule, 1999). For example, the mouse mutation UInaless (UI) affects cis -regulation of 5'Hoxd genes (Spitz et al., 2003). Ectopic proximal Hoxd13 together with reduced Hoxd11 expression causes severe reduction of both zeugopod skeletal elements in limbs of $U /$ mutants embryos (Herault etal., 1997; Peichel etal., 1997). Moreover, Chen et al. (2004) have established that the Gli3 repressor interacts directly with Hoxd proteins to promote digit specification. Therefore, it is possible to assume that the specific genetic interaction of Gli3 with Alx4 impacts on limb bud morphogenesis at the level of Hox protein regulation and function rather than their expression.

\section{Experimental Procedures}

G/i3+/-; $A / \times 4+1$ - double heterozygous mice were inter-crossed to obtain Gli3-/-; $A / x 4-/$ - double mutant embryos. Embryos and mice were genotyped as described by te Welscher et al. (2002b). Day of vaginal plug detection was defined as embryonic day 0.5 . Embryos of gestational days 10.5-11.75 were dissected in PBS, fixed in $4 \%$ paraformaldehyde and processed for whole-mount in situ hybridization using digoxygenin-labelled antisense riboprobes as described by Haramis et al. (1995). Embryos were agematched by determining their somite number (variation \pm 2 somites). To visualize cartilage, embryos of gestational day E12.5 were fixed $5 \%$ TCA and subsequently stained with alcian green to visualize the cartilage. Embryos were cleared in methyl salicylate. Embryos of gestational days older than E14.0 were stained for cartilage and bone using standard alcian blue and alizarin red staining (Zeller etal., 1989). However, either no or only small ossification centres (red) were detected by E14.5 (Fig. 2, 3)

\section{Acknowledgements}

The authors are grateful to V. Portegijs and H. Goedemans for technical assistance and $C$. Mueller-Thompson for help in preparing the manuscript. We thank G. Martin for providing the Sox9 probe and F. Meijlink for helpful discussions and support. This study was supported by the Swiss National Science Foundation (R.Z), the Dutch NWO (R.Z), KNAW (A.Z) and the Stichting Catharine van Tussenbroek (L.P).

\section{References}

AHN, S and JOYNER, A. L. (2004). Dynamic changes in the response of cells to positive hedgehog signaling during mouse limb patterning. Cell 118: 505-516.

BUSCHER, D., BOSSE, B., HEYMER, J. and RUTHER, U. (1997). Evidence for genetic control of Sonic hedgehog by Gli3 in mouse limb development. Mech Dev 62: $175-82$.

CHEN, Y., KNEZEVIC, V., ERVIN, V., HUTSON, R., WARD, Y. and MACKEM, S. (2004). Direct interaction with Hoxd proteins reverses Gli3-repressor function to promote digit formation downstream of Shh. Development 131: 2339-47.

CHIANG, C., LITINGTUNG, Y., HARRIS, M.P., SIMANDL, B.K., LI, Y., BEACHY, P.A. and FALLON, J.F. (2001). Manifestation of the limb prepattern: Limb development in the absence of Sonic Hedgehog function. Dev. Biol. 236: 421-435.

DAVIS, A. P., WITTE, D. P., HSIEH-LI, H. M., POTTER, S. and CAPECCHI, M. (1995). Absence of radius and ulna in mice lacking hoxa-11 and hoxd-11. Nature 375: 791-795.

GOFF, D. J. and TABIN, C. J. (1997). Analysis of Hoxd-13 and Hoxd-11 misexpression in chick limb buds reveals that Hox genes affect both bone condensation and growth. Development 124: 627-636.

HARAMIS, A. G., BROWN, J. M. and ZELLER, R. (1995). The limb deformity mutation disrupts the SHH/FGF-4 feedback loop and regulation of 5'HoxD genes during limb pattern formation. Development 121: 4237-4245.

HARFE B.D., S. P. J., NISSIM S., TIAN H., MCMAHON A.P. and TABIN C.J. (2004). Evidence for an expansion-based temporal Shh gradient in specifying vertebrate digit identities. Cell 118: 517-528.

HERAULT, Y., FRAUDEAU, N., ZAKANY, J. and DUBOULE, D. (1997). Ulnaless (UI), a regulatory mutation inducing both loss-of-function and gain-of-function of posterior Hoxd genes. Development 124: 3493-500.

HUI, C. and JOYNER, A. (1993). A mouse model of greig cephalopolysyndactyly syndrome: the extra-toesJ mutation contains an intragenic deletion of the Gli3 gene. Nat-Genet. 3: 241-246.

JOHNSON, D. R. (1967). Extra-toes: a new mutant gene causing multiple abnormalities in the mouse. J. Embryol. exp. Morph. 17: 543-581.

KRAUS, P., FRAIDENRAICH, D. and LOOMIS, C. A. (2001). Some distal limb structures develop in mice lacking Sonic hedgehog signaling. Mech Dev 100: 4558. 
MACIAS, D., GANAN, Y., RODRIGUEZ-LEON, J., MERINO, R. and HURLE, J. M. (1999). Regulation by members of the transforming growth factor beta superfamily of the digital and interdigital fates of the autopodial limb mesoderm. Cell Tissue Res. 296: 95-102.

MASUYA, H., SAGAI, T., WAKANA, S., MORIWAKI, K. and SHIROISHI, T. (1995). A duplicated zone of polarizing activity in polydactylous mouse mutants. Genes Dev 9: 1645-53.

PEICHEL, C. L., PRABHAKARAN, B. and VOGT, T. F. (1997). The mouse UInaless mutation deregulates posterior HoxD gene expression and alters appendicular patterning. Development 124: 3481-3492.

QU, S., NISWENDER, K., JI, Q., VAN DER MEER, R., KEENY, D., MAGNUSON, M. A. and WISDOM, R. (1997). Polydactyly and ectopic ZPA formation in $A / x-4$ mutant mice. Development 124: 3999-4008.

QU, S., TUCKER, S. C., EHRLICH, J. S., LEVORSE, J. M., FLAHERTY, L. A., WISDOM, R. and VOGT, T. F. (1998). Mutations in mouse Aristaless-like4 cause Strong's Luxoid polydactyly. Development 125: 2711-21.

SCHIMMANG, T., LEMAISTRE, M., VORTKAMP, A. and RÜTHER, U. (1992). Expression of the zinc finger gene Gli3 is affected in the morphogenetic mouse mutant extra-toes (Xt). Development 116: 799-804.

SPITZ, F., GONZALEZ, F. and DUBOULE, D. (2003). A global control region defines a chromosomal regulatory landscape containing the HoxD cluster. Cell 113: 40517.

TAKAHASHI, M., TAMURA, K., BUESCHER, D., MASUYA, H., YONEI-TAMURA, S., MATSUMOTO, K., NAITOH-MATSUO, M., TAKEUCHI, J., OGURA, K., SHIROISHI, T. et al. (1998). The role of $A / x-4$ in the establishment of anteroposterior polarity during vertebrate limb development. Development 125: 4417-4425.

TE WELSCHER, P., FERNANDEZ-TERAN, M., ROS, M. A. and ZELLER, R. (2002a).
Mutual genetic antagonism involving GLI3 and dHAND prepatterns the vertebrate limb bud mesenchyme prior to SHH signaling. Genes Dev 16: 421-426.

TE WELSCHER, P., ZUNIGA, A., KUIJPER, S., DRENTH, T., GOEDEMANS, H. J., MEIJLINK, F. and ZELLER, R. (2002b). Progression of Vertebrate Limb Development through SHH-Mediated Counteraction of GLI3. Science 298: 827-830.

WANG, B., FALLON, J. F. and BEACHY, P. A. (2000). Hedgehog-Regulated Processing of Gli3 Produces an Anterior/Posterior Repressor gradient in the Developing Vertebrate Limb. Cell 100: 423-434.

WRIGHT, E., HARGRAVE, M. R., CHRISTIANSEN, J., COOPER, L., KUN, J., EVANS, T., GANGADHARAN, U., GREENFIELD, A. and KOOPMAN, P. (1995). The Sry-related gene Sox9 is expressed during chondrogenesis in mouse embryos. Nat Genet 9: 15-20.

ZAKANY, J. and DUBOULE, D. (1999). Hox genes in digit development and evolution. Cell Tissue Res. 296: 19-25.

ZELLER, R. (2004). It takes time to make a pinky: unexpected insights into how SHH patterns vertebrate digits. SCI STKE 2004: 53.

ZELLER, R., JACKSON-GRUSBY, L. and LEDER, P. (1989). The limb deformity gene is required for apical ectodermal ridge differentiation and anteroposterior limb pattern formation. Genes and Dev. 3: 1481-1492.

ZUNIGA, A., HARAMIS, A. P., MCMAHON, A. P. and ZELLER, R. (1999). Signal relay by BMP antagonism controls the SHH/FGF4 feedback loop in vertebrate limb buds. Nature 401: 598-602.

ZUNIGA, A. and ZELLER, R. (1999). Gli3 (Xt) and formin (Id) participate in the positioning of the polarising region and control of posterior limb-bud identity. Development 126: 13-21. 